\title{
A NEW DESIGN OF FLICKER PHOTOMETER FOR LABORATORY COLORED LIGHT PHOTOMETRY.
}

By Herbert E. Ives and Edw. J. Brady.

$\Gamma^{\mathrm{T}}$ is now rather generally agreed that the difficulties of colored light photometry are best met in practice by eliminating the color differences through the medium of colored absorbing screens or auxiliary standards of different colors. Given the colored absorbing screens or other means the next matter is to determine their photometric transmissions or candle power values. In this determination all the difficulties of heterochromatic photometry must be faced, and a choice of instruments and photometric methods must be made. Later such choice must be made the subject of general agreement among the standardizing laboratories in whose hands such calibrating work should rest.

From a previous study of the subject ${ }^{1}$ the conclusion has been drawn that the most desirable instrument for this purpose is the flicker photometer, but with the reservation that certain conditions of illumination and field-size must be adhered to and that the average results of many observers must be taken. Reference should be made to the original papers for the details of the work leading to these conclusions. The present paper describes a design of flicker photometer intended for the class of work mentioned and planned to be used in the manner specified.

Since a number of points of importance in the design of any flicker photometer were learned in the previous study, attention is here at the outset given to an account of these, as preliminary to the description of how these points have been provided for in the new instrument.

The attainment of high sensibility in a flicker photometer is dependent upon careful attention to several factors:

First, the dividing line between the two fields which are alternated should be a line without breadth,

Second, the speed of alternation should be accurately adjustable, and

Third, it should be possible to vary the relative brightness of the two fields quickly over a wide range with a minimum of physical effort.

The first point is quite as important in the flicker photometer as in the

1 Philosophical Magazine, July, Sept., Nov., Dec., I9r2, "Studies in the Photometry of Lights of Different Colors," Herbert E. Ives. 
ordinary equality or contrast type. A thick dividing line in the latter instrument reduces the sensibility. In the flicker photometer a black line produces flicker of itself, necessitating a certain speed of alternation merely to make this mechanical imperfection disappear. As noted below, an unnecessary increase of speed decreases sensibility.

The question of speed in a flicker photometer is importani, although the emphasis has often been laid on a mistaken idea. The speed determines the sensibility, and the speed necessary in any particular case is determined by the color difference. The speed does not affect the absolute values obtained, as has been thought by some, but it does change the accuracy of setting. For illustration, in Fig. I let $A B$ be the photometer bar on which a flicker head is movable, having at $A$ and $B$ lights of equal candle power. If the speed of alternation is high enough (say, 70 or 80 alternations per second) the photometer head may be placed anywhere on the bar and no flicker can be perceived; this condition is represented by the line $a-a$. If the speed is now dropped to a certain lower value, it is found that at the ends of the bar flicker is visible, but for a long space in the center one point of setting is as good as another, $(b-b)$. At each successive decrease of speed the region of uncertainty is decreased, hence the sensibility is increased. If the two lights are different in color this decrease of speed may be carried

only so far as no perception of Graphical Representation of Speed-Sensibilcolor alternation appears. The

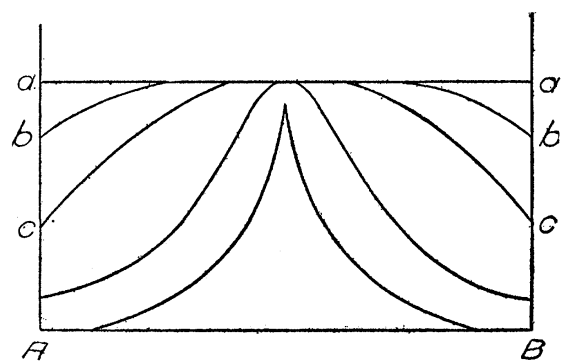

Fig. 1. ity Relations in the Flicker Photometer. greater the color difference the higher must be the speed to prevent "color flicker."

An interesting consequence follows from these considerations, namely, that with no color difference the speed demanded is zero, in other words, that the flicker photometer degenerates into an equality photometer in its limiting case where no color differences exist to call for the fusion due to alternation. Instructions which are sometimes given to the effect that the "correct" speed is to be determined with two lights of the same color and this speed then used with differently colored lights, are misleading, unless the speed called for by a bad dividing line is higher than that demanded by the color difference.

With regard to the third point, it is to be remembered that the position of match is that of minimum of flicker. Were the eye to maintain its 
capacity to perceive flicker absolutely constant from moment to moment, to remain absolutely fixed in position, and were it possible to maintain the speed of alternation exactly the same, then the point of disappearance of flicker might be sought and worked up to slowly from each side. But in practice it is always best to adjust the speed until a very sharp minimum of flicker is produced. In finding a maximum or minimum point it is useless to approach from one side. Instead a series of oscillations about this point is necessary, the oscillations constantly decreasing in amplitude until the slightest touch either way increases the flicker. In order to produce these oscillations of brightness best, they should be producible by the easy motion of the hand or fingers. If a motion of the body is necessary, the eye also is apt to be moved from the photom-

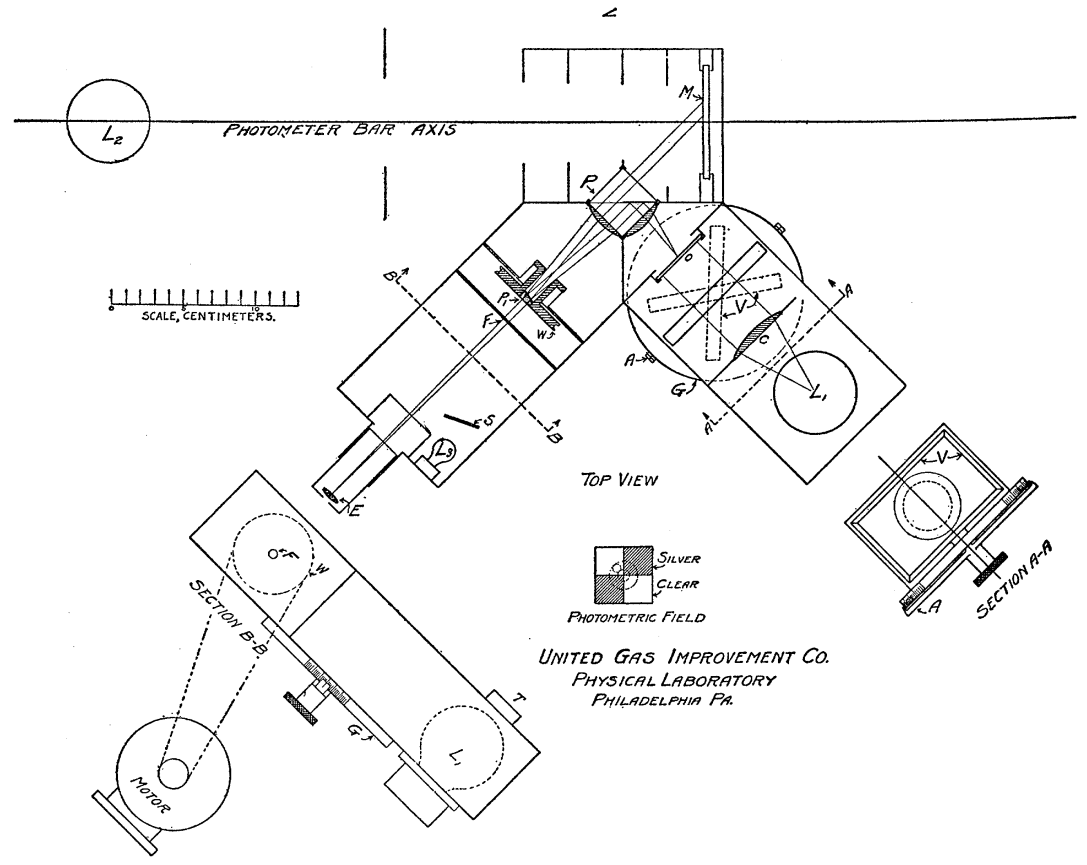

Fig. 2.

eter eye-piece and this movement, even when small, is seriously disturbing. It causes, beside the disturbance of attention, an increase of flicker just as a quickly moving object reveals the fluctuating character of an otherwise apparently steady light. Obviously a flicker photometer head should be of the stationary and not of the movable type.

The instrument is best described by reference to Fig. 2, which gives plan and sections. The photometer is planned to be used solely by the 
substitution method, so that while the test and standard light sources are carried successively at appropriate places on the photometer bar, the comparison lamp is held at a fixed position in the photometer body. Tracing the paths of the beams of light which ultimately reach the eye will be the easiest way to understand the photometer's behavior. The light from the standard lamp $\left(L_{2}\right)$ falls upon the matt white surface $(M)$; thence a portion is reflected through the cube $(P)$ and the small prism $\left(P_{1}\right)$ to the eye. The light from the comparison lamp $\left(L_{1}\right)$ passes through the lens $(C)$; thence through the variable neutral-tint screen $(V)$ to the flashed opal glass $(O)$. Part of the diffused light of the latter enters the cube $(P)$, is reflected through the small prism $\left(P_{1}\right)$ to the eye-piece and eye at $E$.

The mechanism for producing the alternation of the two illuminations is comprised in the cube $(P)$ and the prism $\left(P_{1}\right)$. The cube is a modified Lummer-Brodhun. One of the component prisms is left untouched; the other is silvered and the silvering cut away in the manner shown in the separate sketch of the photometric field. In the present case the silver was cut away with a sand blast, after which the two prisms were cemented together with Canada Balsam, which practically eliminated the diffusing roughness of its surface originally caused by the sand-blast. The slight selective coloring, due to the silver, is of course of no importance, because of the substitution method employed. The small prism $\left(P_{1}\right)$, having an angle of $10^{\circ}$, is mounted in a collar $(W)$, which is rotated by the motor to which it is connected by a belt. The plate $(F)$, perforated by a central round opening of $2^{\circ}$ diameter, as viewed from the eye-piece, limits the actual area of the prism used. When the motor is running the beam of light which reaches the eye is, as it were, whirled around the axis of the instrument, alternating passing over the clear and the silvered parts of the cube $(P)$. The successive appearance of the openings at $F$ are readily seen from the separate sketch of the photometric field, where the small circle represents the section of the beam coming to the eye from $F$. The practically invisible dividing line between the silvered and the clear portion of the cube and the smooth continuous motion of the prism $\left(P_{1}\right)$ insures the first condition considered above.

The second condition above dwelt upon is secured by using a series wound motor with a sliding variable resistance. In conjunction with the latter, sockets are provided in the base of the instrument for the insertion of various fixed resistances; these, together with the variable resistance, provide for any desired speed.

The third desirable condition is that in regard to easy variation of the relative illumination. This is here secured by the use of the variable 
neutral tint screen $(V)$. This consists of two opaque line gratings on glass slightly separated from each other. When rotated about an axis parallel to the lines the transmission ${ }^{1}$ varies with a rapidity determined by the separation of the glasses. This screen is rotated by a milled head which also carries the pointer $(A)$. The size of the milled head is such that it may be turned easily by the fingers. Small lights to illuminate the scale are also provided, but are not shown in the sketch, and the scale may be read either from the observer's side or else by an assistant viewing the other end of the pointer. The lens $(C)$ is intended to help make the illumination on $(O)$ uniform, and as a further refinement the cube $(P)$ is furnished with a convex lens focused on one spot of the opal glass $(O)$. A calibration curve of the screen in this particular

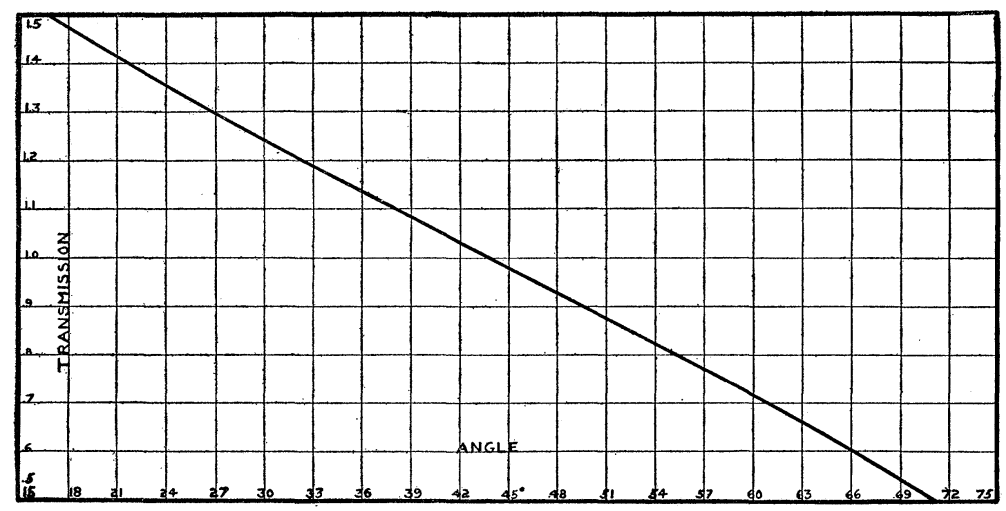

Fig. 3.

Calibration Curve of Variable Neutral Tint Screen.

instrument is shown in Fig. 3. It is practically a straight line over all the region which would be used, remembering that it is intended to have all measurements made at a single brightness (corresponding to 25 meter candles on a white surface), depending upon the variable screen merely for small ranges.

A novelty in this instrument is the means for illuminating the surroundings of the photometric field to nearly the same brightness, furnished by the small lamp $\left(L_{3}\right)$ which throws its light on the white walls of the compartment facing $F . S$ is a small translucent screen for securing uniform illumination. This bright surrounding field materially contributes to the comfort of reading.

Mechanical features of importance are the ventilating openings, of which one is illustrated at $T$, and the care taken to make every optical part immediately removable for cleaning. This latter is a point often neglected by instrument makers.

1 "A Variable Neutral Tint Screen," H. E. Ives, Electrical World, Mar. I6, rgr2. 
The photometer in use thus far has proved itself admirably sensitive. A recent set of readings by two observers furnished the following figures, which are representative: With a very slight color difference between the comparison and test (incandescent electric) lamp the mean variation from the mean of ten settings by observer "A" was .38 per cent. and by observer " $B$ " .45 per cent. With the test light a nearly monochromatic green (equivalent wave-length $.546 \mu$ ), the corresponding figures were .84 per cent. and .67 per cent. A set of seven readings on the same light source, the apparatus having been disturbed for other readings in between, showed an extreme variation from the mean of less than one per cent.

The instrument is now being used to calibrate certain absorbing solutions which have been developed to eliminate the color difference between the more important artificial illuminants and the ordinary carbon lamp standards.

Physical Laboratory, The United Gas Improvement Company, May, rgr2. 